\title{
IN VITRO DEGRADATION AND PHASE COMPOSITION OF COSMETIC EMULSIONS
}

\author{
RAMONA I. CHELCEA ${ }^{a}$, DUMITRITTA MOLDOVAN ${ }^{a}$, DAN E. DEMCO ${ }^{a}$, \\ EUGEN CULEA ${ }^{a}$ AND RADU FECHETE ${ }^{a *}$
}

\begin{abstract}
Microscopic properties like network dynamics and phase compositions were studied for a series of commercially available cosmetics emulsions like hair shampoo, anti-UV shielding creams and hand and body creams. Proton longitudinal and transverse nuclear magnetic relaxations were measured by recording the $1 \mathrm{D}$ CPMG and $2 \mathrm{D}$ saturation recovery-CPMG echoes decays. The one and bi-dimensional inverse Laplace transformations reveals subtitle features which characterize the microscopic dynamic behaviour associated with the in vitro natural degradation of cosmetics. The IR and UV-VIS spectra were recorded and correlated with the NMR measurement.
\end{abstract}

Keywords: commercial cosmetics; NMR; $T_{2}$ relaxation; $T_{1}-T_{2}$ correlation; NMR-MOUSE ${ }^{\circledR}, I R$ and UV-VIS spectroscopy.

\section{INTRODUCTION}

Daily the human body is assaulted by food, cosmetic or pharmaceutical products and can develop a significant sensitivity in interaction with these consumer products. For example N-Nitrosamines are expected to be human carcinogens because of evidence of carcinogenicity in experimental animals [1]. Humans can be exposed to Nitrosamines exogenously and via endogenous formation. Exogenous exposure can occur from a variety of products like: food and drink (beer), inhalation of tobacco smoke, use of rubber products (balloons, teats and soothers) and cosmetics [2-4].

\footnotetext{
a Technical University of Cluj-Napoca, Department of Physics and Chemistry, 25 G. Baritiu Str., RO-400027, Cluj-Napoca, Romania

* Corresponding author: rfechete@phys.utcluj.ro
} 
The stratum corneum $(10-20 \mu \mathrm{m})$ is the most upper layer of the epidermis which together with dermis and subcutaneous fat tissues forms the skin. This is composed of intracellular lipids and dead cells (corneocites - flat cells of keratin-containing structures enveloped in proteins) and has a protective role against environmental aggression, being also a barrier to water loss and foreign body penetration. In stratum corneum (SC) a substantial amount of water is bounded by the keratin and by so called natural moisturizing factors (humectants) like some amino acids, lactate, urea, uric acid, glucosamine, creatine, citrate, formate or ions $\left(\mathrm{Cl}^{-}, \mathrm{Na}^{+}, \mathrm{K}^{+}, \mathrm{Ca}^{2+}, \mathrm{Mg}^{2+}\right)$ etc. The hair fibres are composed from a central cortex enveloped into 6 to 10 layers of overlapping cells named cuticle. In order to prevent degradation of skin or hair a large variety of specialized cosmetics emulsions with multiple targets (cleansers, humectants, emollients, sunscreen and occlusive agents or antipuretic and/or antiacne medicaments) are produced and are commercially available. Thus, for example glycerol and urea are well-known humectants, which are highly water soluble and therefore difficult to be deposited into skin, and petroleum which is a common occlusive agent [5 and references therein].

An emulsion is usually defined as an opaque, heterogeneous system of two immiscible liquid phases where one of the phases is dispersed in the other as drops of microscopic or colloidal size (around $1 \mu \mathrm{m}$ ) and stabilized against separation [6]. Common cosmetic emulsions can be found as: i) oil in water (o/w) - which are the majority; ii) water in oil (w/o); iii) aqueous gel or iv) silicone in water. Thus, many types of silicone can be used to create cosmetics with the appropriate degree of hair conditioning. Among these, the dimethicone copolyols have a light conditioning effect but are largely used due to their reduced eye irritation and to boost the foaming properties of shampoos [5].

Cleanser products are mainly designated to remove unwanted materials (e.g. dirt, oil or sebum) from skin and hair. Unfortunately charged surfactants like anionic (e.g. phosphoric, sulfonic or acyl amino acids and salts) and cationic are aggressive, they can reduce the skin moisture and can increase the skin's irritation. Fortunately, there are other commercially available surfactants (nonionic and amphoteric derivate from anionic variants) that are mild to the skin [5]. Aqueous polymeric dispersions are used extensively as thickeners in cosmetics and pharmaceutical products to improve their rheological properties [7-10]. For example the cross-linked thickeners can form a networked microgel structure in solutions and are also very useful as platforms in drug delivery applications because of a better control release of medicaments [11, 12]. The UV filters (sunscreens) in skin care products bring important benefit to cosmetic formulations. For classification of such products function of the degree of protection, a sun protection factor (SPF) is defined as the dose of UV radiation required to produce 1 minimal erythema dose on protected skin surface on which is applied $2 \mathrm{mg} / \mathrm{cm}^{2}$ of product [5]. 
In the last two decades, strongly inhomogeneous static and radio frequency magnetic fields becomes a prosperous environment for quality NMR techniques to evolve. More and more NMR applications have been proposed and operate successfully in these fields $[13,14]$. These can includes the stray field NMR [15,16], as well as the development of surface NMR spectrometers $[17,18]$ and sensors [19, 20] for material testing [21-24], imaging [25, 26] and well logging [27-29]. Moreover, approaches toward high-resolution ex-situ NMR spectroscopy have been also recently discussed [30, 31].

The present work proposes a study of the cosmetic compounds phases' stability when they are subjected to external natural degradation factors. As example, three cosmetics categories were chosen: hair shampoo, anti-UV shielding creams and hand and body creams (see Table 1).

\section{RESULTS AND DISCUSSION}

The recorded 1D NMR signal, $S_{T_{2}}\left(t_{2}\right)$, the decay of a CPMG echoes trains is analysis by an one-dimensional Laplace inversion algorithm, using Prospa software [32], to extract the transversal relaxation time distribution function $f\left(\mathrm{~T}_{2}\right)$ from,

$$
S_{T_{2}}\left(t_{2}\right)=\int f\left(T_{2}\right) \exp \left\{-\frac{t_{2}}{T_{2}}\right\} d \mathrm{~T}_{2} .
$$

A cosmetic product usually consists of a large number of ingredients which can interact with each other forming a smaller number of phases. In the following we will name an emulsion phase that component of the sample which presents a similar microscopic network dynamic, quantified in our experiments with a similar value of relaxation time. In time, during the use or conservation, these phases can present various changes leading to an altered product with modified properties. The study of phase alteration, via the changes in the transverse relaxation times distributions in controlled laboratory conditions to simulate the daily use (up to $24 \mathrm{~h}$ ), is presented in Fig. 1, for the series of cosmetic samples listed in Table 1. The cosmetic samples used in this study can be grouped in three categories: i) hair shampoos; ii) anti-UV shielding creams and iii) hand and body creams.

In the analysis of such $T_{2}$ distributions the main interest is on the peak position (changes of peaks positions) and area under the peaks. Then the peaks located at largest values of $T_{2}$ time can be associated with a low viscous 
emulsion phase or volatile component while the low $T_{2}$ value peak can be associated with high viscous emulsion phase characterized by a more restricted molecular motion. In time, we are expecting to observe on cosmetic samples two opposite effects: i) the loose of moisture by water and volatile components (like perfumes) evaporation and ii) to increase of water amount for the cosmetic sample containing hygroscopic components like glycerin. The area under the peaks which is proportional with the number of ${ }^{1} \mathrm{H}$ is a good indication of the amount of each component in the measured sample.

The shampoos and anti-UV creams presents, at least initially, three main $T_{2}$ peaks (see Figs 1a-1d). The time-dependent $T_{2}$ distributions for both shampoos and anti UV shielding cream with SPF 15 (Figs 1a-1c) are shifted towards smallest $T_{2}$ values, becoming in this way more viscous. This is due probably by losing water, since in shampoos the amount of volatile components like perfumes is small. For these samples the most viscous component (characterize by a small $T_{2}$ ) becomes in time more rigid and looks for both hair shampoos (see Figs 1a and 1b) to have the same area; while the middle and largest $T_{2}$ component seems to suffer an interaction process, more visible for hair shampoo 1 (Fig. 1a).

Thus, for this sample, after approximately 10 hours, the largest $T_{2^{-}}$ component became smaller and is moved toward the middle peak. Contrary, the hair shampoo 2 after approximately 6 hours looks to present an exchange between the middle and the high $T_{2}$-value peaks, both becoming closer and higher, probably on account of rigid component.

It is of interest to compare the dynamic behavior of the two anti-UV shielding creams with standard protection factor, SPF 15 and 25. The most mobile components (large $T_{2}$-value peak), appears to not be too much affected. Since the anti-UV creams have also the role to hydrate the skin, and assuming that the moisture is associated with the more mobile component (or high $T_{2^{-}}$ value peak) then is perfectly reasonable that this component to be less affected in time. The behavior of the other two components is completely different. The middle peak of the SPF 15 cream remain unchanged for almost 10 hours when starts to merge with the rigid component (see Fig 1c). The last one, at the beginning is increasing in rigidity (observed as a decrease of the $T_{2}$ value) but then after 5-6 hours start to became more and more mobile. Contrary, for the SPF 25 cream, in a time of 3 hours, the left peak (more rigid component - small $T_{2}$ value) and middle peak merges with the right peak (most mobile component), excepting a small residue component at $T_{2}$ value around $0.01 \mathrm{~s}$ (see Fig. $1 \mathrm{~d}$ ). 
a)

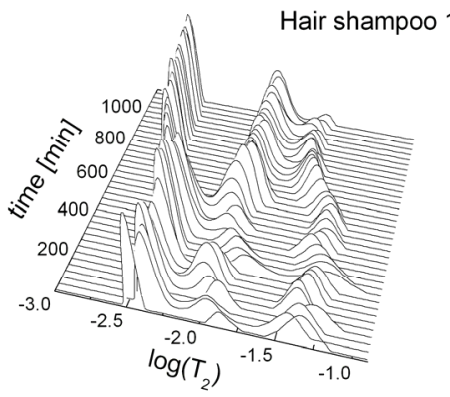

c)

Anti UV shielding cream SPF 15

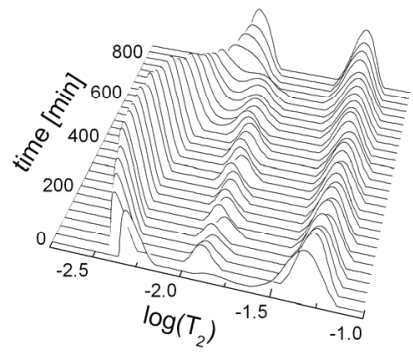

e)

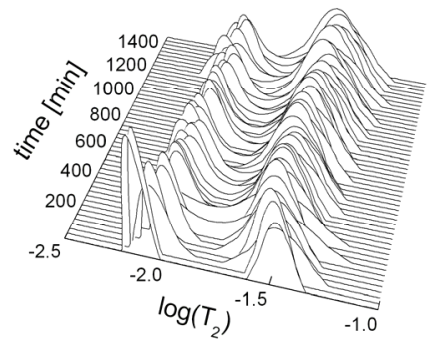

g)

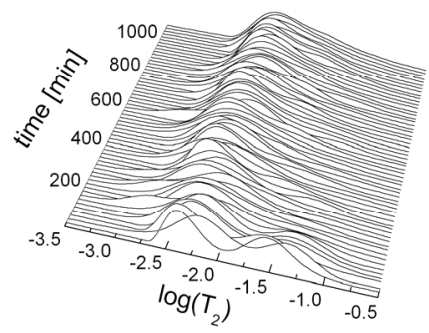

b)
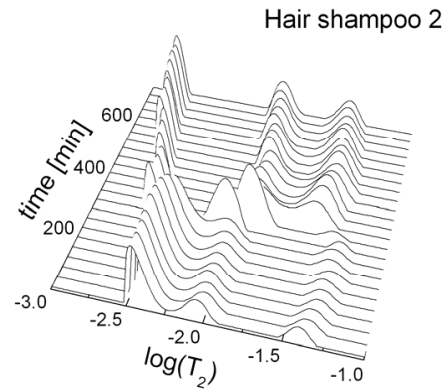

d)

Anti UV shielding cream SPF 25

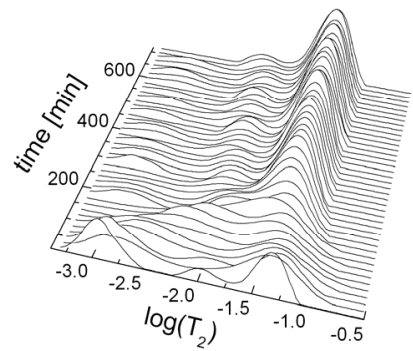

f)

Hand cream 2

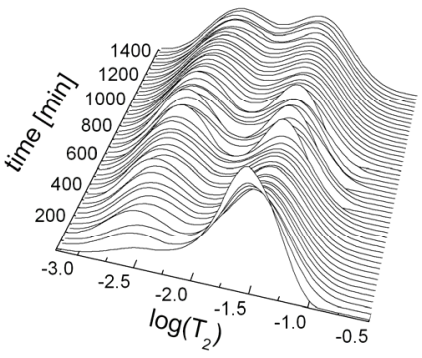

h)

Body cream

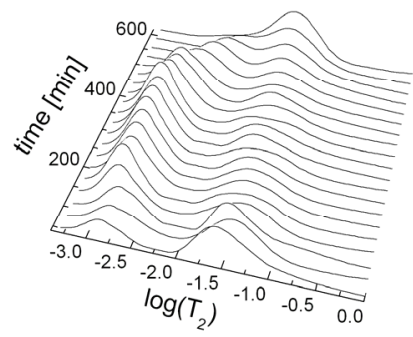

Figure 1. The time dependent normalized probability distribution functions of $\log \left(T_{2}\right)$ for a series of commercial samples like: two hair shampoos a) and b) two anti-UV shielding creams c) and d); three hand creams e), f) and g); and one body cream $\mathrm{h}$ ). 
The dynamics reflected in the time evolution of recorded Laplace spectra is different for those three hand cream samples (Fig. 1e-1g). All three samples present only two components, which can be differentiated by their dynamics by the two peaks observed in their $T_{2}$-distribution functions. While, for the hand cream 1, the dynamic phases can be clearly differentiated, for the hand cream 2 and 3 we cannot distinguish between these phases. No spectacular changes in time are to be reported. In the experimental and data analysis errors, the hand cream 1 seems to be unaffected (see Fig. 1e). A small transitory process, up to 2-3 hours, can be identified for the hand cream 2, were the amount of mobile component percentage decrease with the increase of the rigid component amount (see Fig. 1f). A different, but fast, transitory process can be observed for hand cream 3 where only in the initial time regime a mobile peak is observed (see Fig. 1g). This became very fast so small that is hidden by the more broaden middle peak, which covers several orders of magnitude in the $T_{2}$-distribution. This is an indication of a sample with heterogeneous dynamic components, but stabile in time. In fact all hand creams can be characterized as stabile in the measurement time window.

The body cream presents also two dynamic components (see Fig. 1h). As a particularity, for this sample one can observe a component with a restricted dynamics compared with the rest of cosmetic samples characterized by a $T_{2}$ value around $1 \mathrm{~ms}$. After a transitory regime of several hours, in which this peak increase in amplitude without a change in position, then starts to decrease and to be shifted toward larger $T_{2}$-values, indicating an increase in mobility. Finally, both peaks merge into a single dynamic component with the characteristics of the initial mobile phase.

Up to this point we were able to identify two or three components but we estimate that the $T_{2}$ measurements alone are generally insufficient to distinguish and associate entirely the phase components of studied cosmetics samples. For a better characterization of our colloids we need a new approach, based on the correlation of $T_{1}-T_{2}$ relaxation times. Here, the 2D NMR measured signal, $S_{\mathrm{T}_{1}, \mathrm{~T}_{2}}\left(\tau_{1}, \mathrm{t}_{2}\right)$ is function of $\left.\mathrm{i}\right)$ the measurement parameters (see the pulse sequence from experimental and sample section) like the magnetization recovery time, $\tau_{1}$ and total echo time $t_{2}\left(\tau_{2}+\tau_{2}^{\prime}\right)$ starting after excitation pulses and ii) depends on the sample parameters like longitudinal, $T_{1}$ and transversal, $T_{2}$ relaxation times, respectively [33],

$$
S_{T_{1}, T_{2}}\left(\tau_{1}, \mathrm{t}_{2}\right)=\iint \mathrm{f}\left(T_{1}, T_{2}\right)\left(1-\exp \left\{-\frac{\tau_{1}}{T_{1}}\right\}\right) \exp \left\{-\frac{\mathrm{t}_{2}}{T_{2}}\right\} d T_{1} d T_{2},
$$

where $f\left(T_{1}, T_{2}\right)$ is the $T_{1}-T_{2}$ distribution function. 
The measured $T_{1}-T_{2}$ correlation maps for the series of sample listed in Table 1 are presented in Fig. 2. Many more dynamic phases can be observed in these distribution functions compared with the 1D $T_{2}$-distributions. The peak projections in the $T_{2}$ dimension correspond with the range of similar peaks presented in Fig. 1, however the 2D extension in the $T_{1}$ domain can reveal particular characteristics. As general features we can observe: i) well defined peaks around $T_{1}=0.1 \mathrm{~s}$ or between $T_{1}=0.1-1 \mathrm{~s}$; ii) excepting two cases two large peaks extended over several order of magnitude in both $T_{1}$ and $T_{2}$; iii) the large distribution at largest value of $T_{1}$ is due to the lack of sufficient data points to describe a complete relaxation, therefore, we will consider only the peak maximum of these distributions. The dashed diagonal line indicates the $T_{1}=T_{2}$ values and the thin diagonal lines indicated the constant $T_{1} / T_{2}$ ratios of 10,100 and 1000 . The peaks with the $T_{1} / T_{2}$ ratios closed to 1 can be associated with aqueous phases and those with large $T_{1} / T_{2}$ ratio can be associated with more viscous phases. Moreover, for all samples, the phases for which the $T_{2}$ relaxation is significantly faster than $T_{1}$ relaxation indicate an exchange of molecules which contain protons, possibly in majority, water [33].

There are several features that must be underlined. The anti-UV cream with SPF 25 (see Fig. 2d) present the sharpest peaks and less extended. Only in this case the maximum $T_{1}$ value is around $1 \mathrm{~s}$. In contrast, the hand cream 2 has the largest $T_{1}$ values around $100 \mathrm{~s}$ (see Fig. 2f).

Sharp distributions presents also the hand cream 1 located close to the $T_{1} / T_{2}=10$ ratio (see Fig. $2 \mathrm{e}$ ). In half of the cases there are some components into a so called forbidden region where $T_{1}<T_{2}$. If these peaks are real, then this can be a consequence of proton exchange between different dynamic components. The $T_{1}-T_{2}$ correlation maps were found to be an important source of information but we steel need information for a better interpretations and peak assignment. Additional spectroscopic measurements were performed into attempt to identify the phase compositions and physicchemical properties.

The FT/IR spectra of the series of cosmetics samples listed in Table 1 are presented in Fig. 3 together with the water spectrum. Water presents three main peaks (Fig. 3 sample W): i) a main asymmetric and broad (3000$3700 \mathrm{~cm}^{-1}$ ) peak centred at $\sim 3450 \mathrm{~cm}^{-1}$ (due to the $\mathrm{O}-\mathrm{H}$ bond); ii) a narrow peak centred at $\sim 1600 \mathrm{~cm}^{-1}$ and iii) a broad band at wavenumber smaller than $1000 \mathrm{~cm}^{-1}$. Excepting some features, all these spectra are similar to water spectra. This is an indication of the large amount of water that can form the aqueous phases of studied cosmetics. The particular features presented by all spectra are smallest, indicating a smallest concentration in the sample product. 

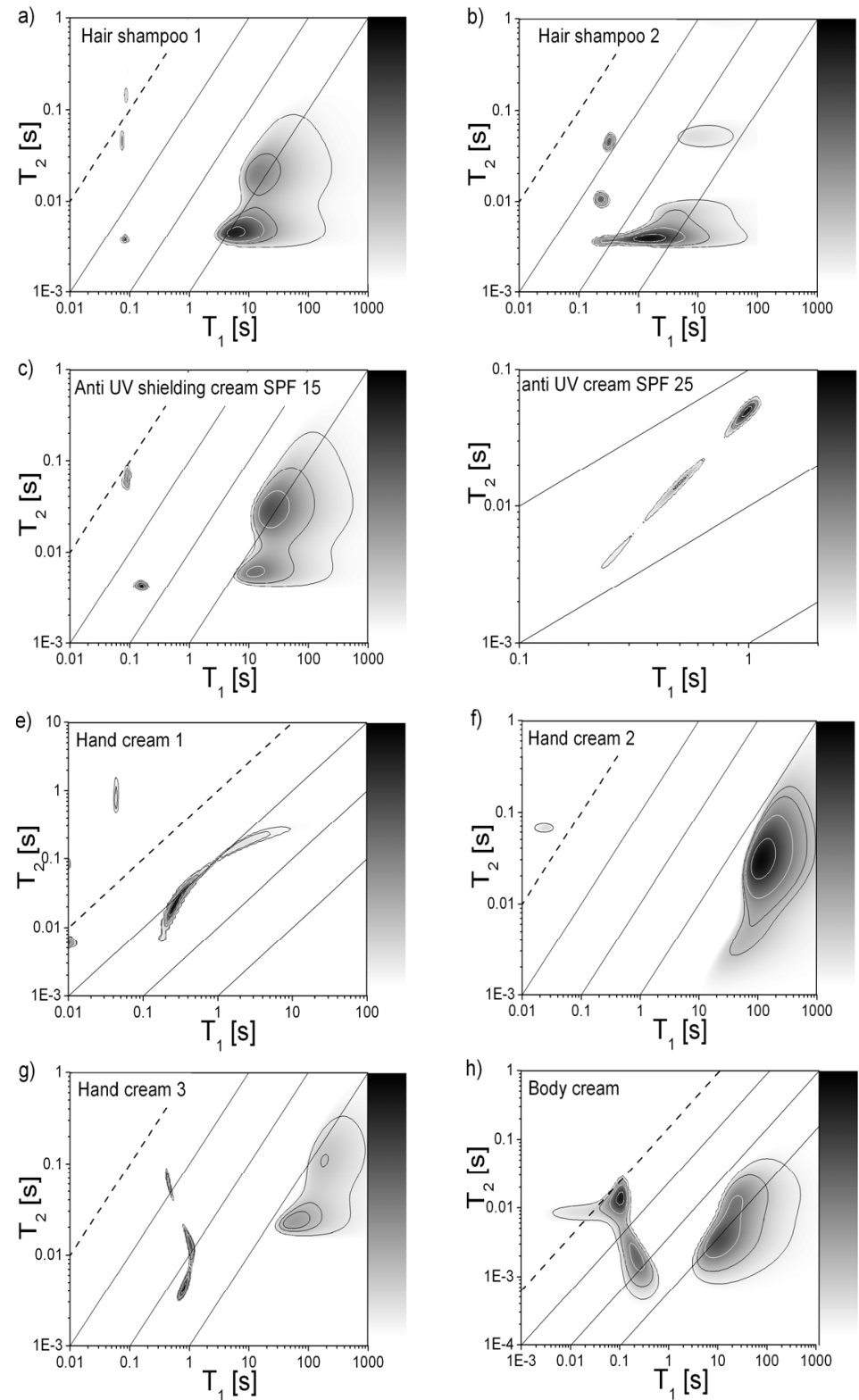

Figure 2. The $T_{1}-T_{2}$ correlation maps for a series of commercial cosmetic emulsions like: two hair shampoos (a) and (b) two anti-UV shielding creams with (c) SPF 15 and (d) SPF 25; three hand creams (e), (f) and (g); and one body cream $(h)$. 
The viscous emulsion dynamics is manifested at lower wave number $1000-1600 \mathrm{~cm}^{-1}$ associated with the skeleton vibration of entire molecule (the fingerprint region) and at large wave number from $2800-3700 \mathrm{~cm}^{-1}$ associated with group characteristic frequencies. Here a broad peak centred $\sim 3200 \mathrm{~cm}^{-1}$ appears as a right shoulder on the water peak, overlapping on this peak, therefore for the majority of samples is identifiable only by comparison with the measured water IR spectra.

All spectra present two relatively large peaks at $\sim 2850 \mathrm{~cm}^{-1}$ and $2925 \mathrm{~cm}^{-1}$ and other left shoulders around them. These can be associated, for example with stearic acid, citric acid, glycerol or other similar ingredients. Many of these compounds present IR peaks between $2800-3000 \mathrm{~cm}^{-1}$ and from $1000-1500 \mathrm{~cm}^{-1}$ and less, then positive final identifications are not possible. Nevertheless, comparing the database ATR-IR spectra of glycerol and paraffin [34] one can identify specific bands (doublet at 2880 and $2934 \mathrm{~cm}^{-1}$ ) of $-\mathrm{C}-\mathrm{H}$ (stretching) bonds and $\sim 1500 \mathrm{~cm}^{-1}$, in our measured ATR-TF/IR spectra.

The anti-UV shielding cream with SPF 15 labelled with C, present a particular peak as a left shoulder on the water peak around $1750 \mathrm{~cm}^{-1}$ may be an indication of a carbonyl compound $(\mathrm{C}=\mathrm{O})$. Moreover, the small peaks at $\sim 1560 \mathrm{~cm}^{-1}$ observed for the samples labelled with C and D (SPF 15 and 25) may be an indication of the presence of aromatic compounds in the antiUV shielding creams. The same indices can be found also for the measured hand cream 1 (see the ATR-FT/IR spectrum labelled with E in Fig. 3). Amines

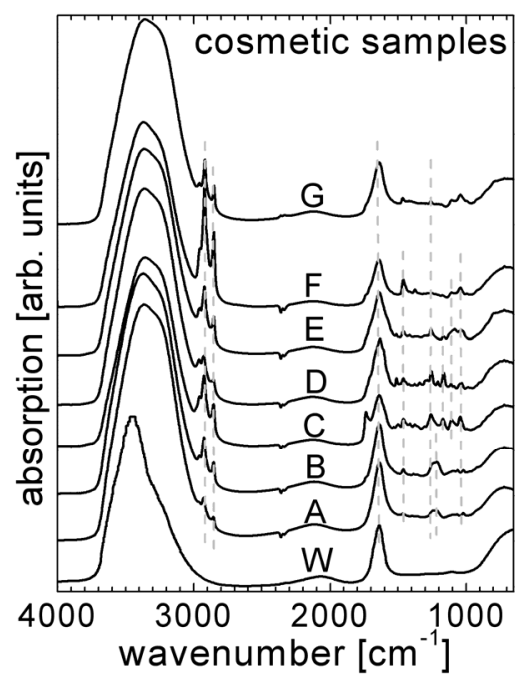

Figure 3. Compared ATR (Attenuated Total Reflection) FT/IR absorption spectra of the series of cosmetics presented in Table 1 together with water (W) IR spectrum. 
(C-N bonds) and alcohols (C-O bonds) functional groups can be found as components in samples presenting peaks between $1030-1230 \mathrm{~cm}^{-1}$ and $1050-1150 \mathrm{~cm}^{-1}$, respectively.

Compared with the time dependent 1D $T_{2}$ distributions (Figs. 1) and 2D $T_{1}-T_{2}$ correlation maps (Figs. 2) ${ }^{1} \mathrm{H}$ NMR data the ATR-FT/IR spectra are more similar. In these spectra the major features are appear grace at the presence of water which for all our samples is found into an amount larger than $50 \%$ (curve W compared with the rest of spectra in Fig. 3). Such amount of water in the cosmetic sample means that the water has to be distributed into mobile phases, characterized by large $T_{2}$ values, but has to be found also in less mobile phases (characterized by smaller $T_{2}$ values) as bound water.

The transmission/absorption characteristics in ultraviolet and visible domains of the distilled colloidal samples are presented in Fig. 4, for comparison together with the UV-VIS water spectra. These present a large absorption peak around $970 \mathrm{~nm}$ and began to be absorbed in the UV domain starting with $300 \mathrm{~nm}$. In addition to water, the cosmetic samples present a continuously increased absorption with no significant features in the visible domain when moving to small wavelength. Starting with $\sim 450 \mathrm{~nm}$ for anti-UV shielding cream with SPF 15 (sample C), $\sim 400 \mathrm{~nm}$ for hand cream 1 (sample E) and $\sim 350 \mathrm{~nm}$ for hair shampoo 2 (sample B) the samples present broadens and features absorption characteristics in the UV domain. Such characteristics may be associated with the presence of paraffin (of which UV characteristic absorption is manifested for wavelength smaller than 350-400 nm) rather than

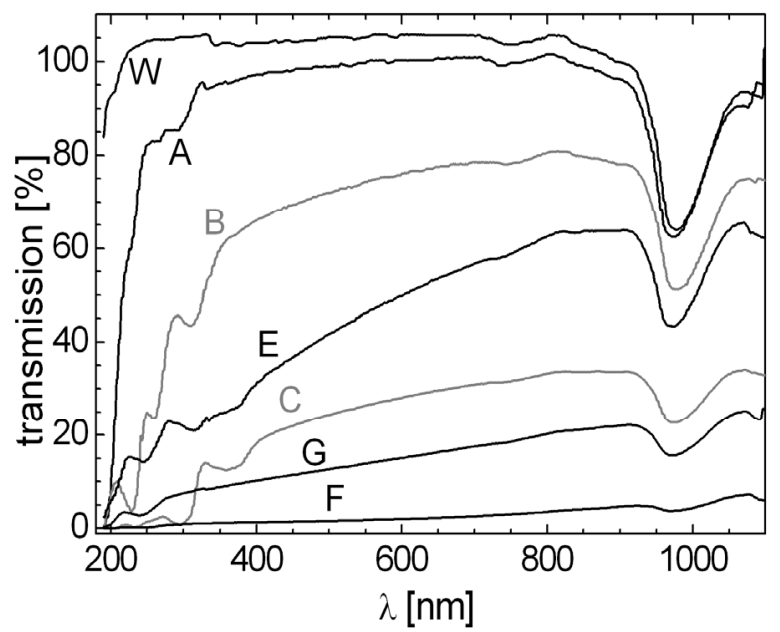

Figure 4. Compared UV-VIS transmission spectra of the series of cosmetics presented in Table 1 and compared with water (W). 
glycerol which present absorption bands up to $550 \mathrm{~nm}$. Diverse features observed as unresolved shoulders in this domain suggest the existence of different ingredients that can absorb the UV radiation, which are in concordance with their use especially the anti-UV creams. The shampoos emulsions present the largest transmission characteristics in near UV and VIS domains.

Contrary to the ATR-FT/IR spectra (Fig. 3), for which the main features are given by the water content, the main features of the UV-VIS spectra (Fig. 4) and of the ${ }^{1} \mathrm{H}$ NMR 1D $T_{2}$ distributions (Fig. 1) and 2D $T_{1}-T_{2}$ correlation maps (Fig. 2) are due to the rest of the components (which for many cosmetic samples the number of this components is larger than 20). Moreover, we can observe that the hair shampoos (samples A and B) with the highest transmittance in UV-VIS spectra (Fig. 4) presents initially (few minutes after the application of the thin film) three balanced peaks in the $T_{2}$ distributions (Figs. 1a and 2b) associated with three dynamic phases. Three $T_{2}$ peaks are observed in the initial regime also for the anti-UV creams (Figs 1d and 1e) and a medium transmittance was measured in the UV-VIS spectra (sample C in Fig. 4). Contrary the samples with the lower transmittance in UV and visible light (as measured from UV-VIS spectra - samples F and G in Fig. 4) presents a quasi-two component $T_{2}$ distribution (poorly resolved distributions - Figs. If and $1 \mathrm{~g}$ ). Probably, the large phase heterogeneity (wide $T_{2}$ distributions) can be correlated with large distributions of electrons levels in cosmetic molecules which will lead to a sample more absorptive in UV-VIS region of the electromagnetic domain.

\section{CONCLUSIONS}

Low field NMR, originally developed to characterize the fluid filled porous media and well-logging, can play now an important role in the characterization, monitoring and quality control of cosmetic products. When accompanied by the new algorithms of Laplace inversion the NMR measurements are a reach source of information about colloidal materials. Thus, the combination of $1 \mathrm{D}$ and $2 \mathrm{D}$ NMR methods that involve transversal and longitudinal relaxation and Laplace inversion data analysis together with IR and UV-VIS spectroscopy was found to be a useful new tool in the characterization of various commercially available cosmetic products. The time dependent $T_{2}$ relaxation distribution functions, which are sensitive to structural composition, texture mobility and interactions of various emulsion components, provided useful information's about cosmetic's phases stability: i) non-stable like the shampoos sample; ii) very stable like hand and body creams or iii) with good hydrating properties like anti UV shielding creams. 


\section{EXPERIMENTAL AND SAMPLE}

The one-dimensional (1D) and two-dimensional (2D) Nuclear Magnetic Resonance relaxometry correlated with advance data processing by the 1D and 2D Laplace inversions and IR and UV-VIS spectroscopy are methods largely used in the study of this class of materials. An appropriate NMR experimental set-up uses a unilateral NMR sensor, the NMR-MOUSE ${ }^{\circledR}$ $[13,14]$. In this case (in contrast with the classical in tube measurement) the tested sample can be subjected to similar conditions as in the case of ordinary use of a thin layer applied on a large surface and with the opposite surface, in contact with the air.

An important tool in highly inhomogeneous magnetic fields NMR experiments is the combination of dedicated pulses sequences like CPMG, which can refocus the linear spin inhomogeneities, with the signal processing by Laplace inversion [13, 14, 27, 29, 32, 33, 35-38]. An elaborate study was performed by Hürlimann et al., on the characterization of food products by twodimensional $D-T_{2}$ and $T_{1}-T_{2}$ distribution function obtained by Laplace inversion [33]. These functions exhibits also distinct components associated there with aqueous and liquid fat content phases. An important step was made by Marigheto et al. by giving several methods for peak assignment in complex $T_{1}-T_{2}$ maps of low-resolution 2D NMR cross-correlation relaxometry [36].

For the NMR measurement a BRUKER Minispec spectrometer with a unilateral NMR-MOUSE ${ }^{\circledR}$ sensor working at $19.2 \mathrm{MHz}$ frequency, was used $[20,37]$. The cosmetic samples were placed directly on the top of the NMR sensor coil on a laboratory ultra-thin glass $(0.1 \mathrm{~mm})$ as a film of $3 \mathrm{~mm}$ thick, covering the entire surface of the radio-frequency coil. For the reproducibility a perforated stencil was used as holder, removed during the measurements. In order to simulate better the contact between the cosmetic sample and the human body the temperature was increased and kept constant at $32^{\circ} \mathrm{C}$. The atmospheric pressure of $990 \mathrm{~mm} \mathrm{Hg}$ and $60 \%$ relative humidity was also monitored.

The molecular dynamics and phase composition of a thin film of emulsion samples subjected to one day degradation in natural conditions was studied by applying the CPMG pulse sequences and recording the spin system response, see Fig. 5 up [37]. A total number of 1000 echoes were recorded with $2 s$ recycle delay and 512 scans to improve the signal to noise ratio. The duration of excitation pulse and refocusing pulses were $5 \mu$ s too.

\footnotetext{
${ }^{\circledR}$ NMR-MOUSE is a registered trademark of RWTH-Aachen.
} 
Preparation

\section{Detection}

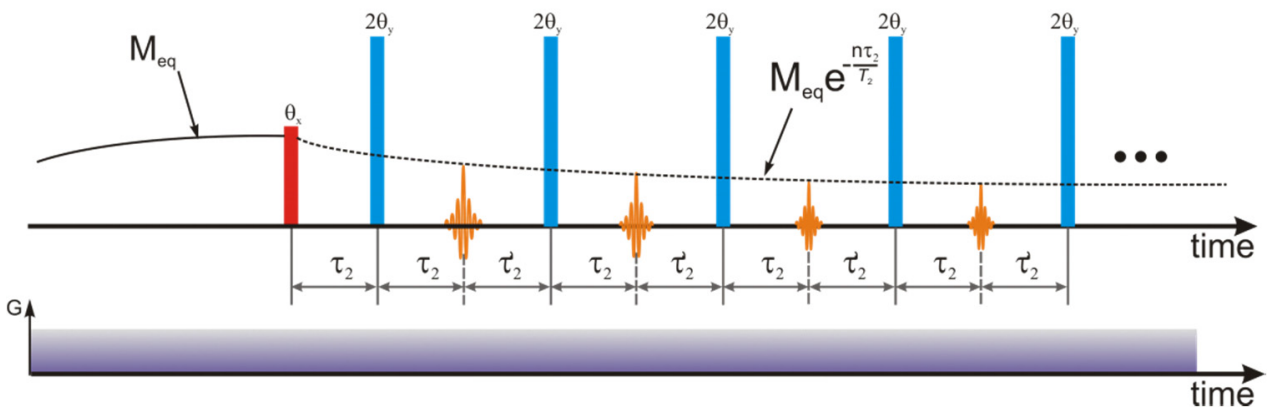

Saturation Recovery

Detection

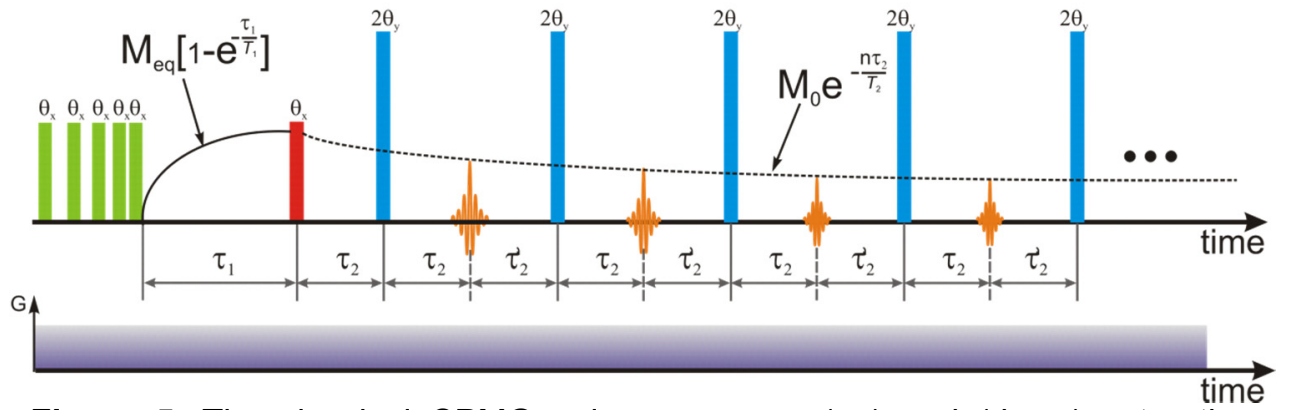

Figure 5. The classical CPMG pulse sequence (up) and (down) saturation recovery followed by detection via CPMG pulse sequence designated for recording the $T_{1}-T_{2}$ correlations maps.

The 2D, $T_{1}-T_{2}$ correlations maps were obtained by recording the spin system response during the CPMG that follow a saturation-recovery pulse sequence with re-magnetization time, $\tau_{1}$, (see Fig. 5 down) [37]. The echo time, $\tau_{2}+\tau_{2}^{\prime}$ was between $100-300 \mu \mathrm{s}$, in function of the measured sample, while the $\tau_{1}$ time was increased in equal steps with $100 \mathrm{~ms}$ up to 5-6 s. For the NMR-MOUSE ${ }^{\circledR}$ sensor, the static magnetic fields gradient across the sample is higher than the strength of the radiofrequency magnetic field. Therefore, all pulses are slice selective $(\sim 0.2 \mathrm{~mm}$ per slice) leading to an initial transient regime in CMPG [33,37]. In order to avoid this effect to affect the $T_{2}$-distribution, the first $10 \mathrm{CPMG}$ echoes were eliminated. The NMR measurements were analyzed using the Prospa software for inverse Laplace transform [32].

The absorption IR spectra were recorded using a single beam FT/IR Jasco 6200 spectrometer with Fourier Transform and an ATR (Attenuated Total Reflection) device. The samples were placed into a $10 \mathrm{~cm}$ long, $5 \mathrm{~mm}$ large and $3 \mathrm{~mm}$ deep holder on top of a special crystal accessory dedicated 
to measurement of liquid samples. The ATR correction procedure was applied in order to obtain similar spectra with those obtained by transmission spectroscopy. Finally, a base line correction procedure was applied. The signal to noise ratio was 16 times improved by recording 256 scans for each sample. The resolution of recorded spectra was $1.928 \mathrm{~cm}^{-1}$ and a full range from $349.05 \mathrm{~cm}^{-1}$ to $4002.57 \mathrm{~cm}^{-1}$.

The UV-VIS spectra were recorded using a single beam CAMSPEC M501 UV-VIS spectrophotometer, and a quartz cell of $1 \mathrm{~cm}$ path length. Due to a high absorbance a smallest droplet of cosmetic samples was mixed with distilled water obtaining solutions with the ratio of emulsion/distilled water presented in Table 1. As an illustration, we present measurements for a variety of cosmetics samples that includes two types of hair shampoo, antisolar ultraviolet radiation creams with different standard protection factor, SPF of 15 and 25, three types of hand creams and one body cream. All these products are commonly commercially available, belong to different cosmetics producers and were purchased from the local specific market. The observed cosmetics present dispersed into dispersion phases with similar or different network dynamics according to i) their compositions, common components like water, glycerol, liquid paraffin or particular components like perfumes and ii) most important, the interactions between these components. For example, glycerin is a neutral, sweet-tasting, colorless, thick liquid which freezes to a gummy paste and which has a high boiling point and can be dissolved into water or alcohol, but not oils. On the other hand, many things will dissolve easier into glycerin so can be characterized as a good solvent. Glycerin is also highly hygroscopic, which means that it absorbs water from the air, an important characteristic for moistening the cosmetics [5].

Table 1. The investigated cosmetic samples: name, associated label and emulsion/distilled-water ratio for UV-VIS measurements.

\begin{tabular}{lcc}
\hline \hline \multicolumn{1}{c}{ Sample Name } & Sample Label & $\begin{array}{c}\text { emulsion/distilled-water } \\
{[\mathrm{mg} / \mathrm{ml}]}\end{array}$ \\
\hline Hair shampoo 1 & $\mathrm{A}$ & 3.56 \\
Hair shampoo 2 & $\mathrm{B}$ & 1.66 \\
Anti UV shielding cream SPF 15 & $\mathrm{C}$ & 1.53 \\
Anti UV shielding cream SPF 25 & $\mathrm{D}$ & - \\
Hand cream 1 & $\mathrm{E}$ & 0.93 \\
Hand cream 2 & $\mathrm{F}$ & 3.56 \\
Hand cream 3 & $\mathrm{G}$ & 1.33 \\
Body cream & $\mathrm{H}$ & - \\
\hline \hline
\end{tabular}




\section{ACKNOWLEDGMENTS}

This work was supported by CNCSIS -UEFISCDI, project number PN II - IDEI code 307/2011. R.F. would like to thank Prof. Dr. B. Blümich for useful dissections related to NMR-MOUSE applications.

\section{REFERENCES}

1. Report on Carcinogens U.S. Department of Health and Human Services, 10th edn. Public Health Service, National Toxicology Program, 2002.

2. G. Ellen, In: Eisenbrand G, Bozler G, Nicolai HV (eds) The significance of Nnitrosation of Drugs. Gustav Fischer Verlag, Stuttgart, 1997, 19-46.

3. Ellen G, Egmond E, van Loon JW, Sahertian ET, Tolsma K, Food Addit Contam, 1990, 7, 207-221.

4. Stephany RW, Schuller PL, Oncology, 1980, 37, 203-210.

5. A.O. Barel, M. Paye, H.I. Maibach, Informa Healthcare USA, New York, 2009 (and references therein).

6. Bernard P. Binks, Modern aspects of Emulsion Science, The Royal Society of Chemistry, Cambridge, 1998.

7. Gary E. LeGrow, W. Leonard Terry, US Patent No 6, 2002, 351, 867.

8. M. Sjoberg, L. Bergstrom, A. Larsson, and E. Sjostrom. Colloids Surf. A, Physicochemical Eng. Asp., 1999, 159, 197-208.

9. K.D. Bremecker, B. Koch, W. Krause, and L. Neuenroth. Pharm. Ind., 1992, 54, 182.

10. J.Y. Kim, J.Y. Song, E.J. Lee, and S.K. Park. Colloid and Polymer Science, 2003, 281, 614 .

11. M. Yonese. In Y. Osada and K. Kajiwara (eds.), Gels Handbook. Vol. 3, Academic Press, San Diego, CA, 2001, 230-240.

12. S. Ding, Pharmaceutical. Science. Technology Today, 1998, 1, 328.

13. N. Cioica, R. Fechete, R. Chelcea, C. Cota, M. Todica, C.V. Pop, O. Cozar, Studia UBB Chemia, 2015, 60 (1), 45-55.

14. D. Moldovan, M. Pop, A. Baudouine, M. Todica, Studia UBB Chemia, 2011, 56 (3) 103.

15. F. Balibanu, K. Hialu, R. Eynael, D.E. Demco, B. Blümich, Journal of Magnetic Resonance, 2000, 145, 246.

16. P.J. McDonald, Progres in Nuclear Magnetic Resonance Spectroscopy, 1997, 30, 69 (and references therein).

17. R.L. Klinberg, A. Sezginer, D.D. Griffin, M. Fukuhara, Journal of Magnetic Resonance, 1992, 97, 466-485.

18. G. Eidmann, R. Savelsberg, P. Blümler, B. Blümich, Journal of Magnetic Resonance. A., 1992, 122, 104-109. 
19. B. Blümich, V. Anferov, S. Anferova, M. Klein, R. Fechete, M. Adams, F. Casanova, Magnetic Resonance Engineering, 2002, 15(4), 255.

20. B. Blümich, V. Anferov, S. Anferova, M. Klein, R. Fechete, Macromolecular Materials Engineering, 2003, 288(4), 312.

21. G. Guthausen, A. Guthausen, F. Balibanu, R. Eymael, K. Hailu, U. Schmitz, and B. Blümich. Macromolecular Materials Engineering, 2000, 276/277, 25.

22. K. Hailu, R. Fechete, D.E. Demco B. Blümich, Solid State Nuclear Magnetic Resonance, 2002, 22, 327.

23. N.O. Goga, A. Pirnau, L. Szabo, R. Smeets, D. Riediger, O. Cozar, B. Blumich, Journal Optoelectronic Advanced Materials, 2006, 8(4), 1430.

24. M. Todica, Journal Optoelectronic Advanced Materials, 2007, 9(3), 633.

25. H. Heise, D. Sakellariou, C.A. Meriles, A.J. Moulé, Journal of Magnetic Resonance, 2001, 156, 146.

26. B. Blümich, F. Casanova, J. Perlo, S. Anferova, V. Anferov, K. Kremer, N. Goga, K. Kupferschläger, M. Adams, Magnetic Resonance Imaging, 2005, 23, 197-201.

27. G. Goelman, and M.G. Prammer, Journal of Magnetic Resonance. A., 1995, 113, 11.

28. Y.Q. Song, L. Venkataramanan, M.D. Hürlimann, M. Flaum, P. Frulla, and C. Straley, Journal of Magnetic Resonance, 2002, 154, 261.

29. M.D. Hürlimann, M. Flaum, L. Venkataramanan, C. Flaum, R. Freedman, G.J. Hirasaki, Magnetic Resonance Imaging, 2003, 21, 305.

30. C.A. Meriles, D. Sakellariou, H. Heise, A.J. Moulé, A. Pines, Science, 2001, 293, 82-85.

31. J. Perlo, F. Casanova, B. Blümich, Science, 2007, 315, 1110 - 1112.

32. P.T. Callaghan, Translational Dynamics and Magnetic Resonance, 2011, Oxford University Press.

33. M. Hürlimann, L. Burcaw, Y.Q. Song, Journal of Colloid Interface Science, 2006, 297, 303.

34. S. Pasieczna-Patkowska, T. Olejnik, Annales Universitatis Mariae Curie-Skłodowska Lublin - Polonia, 2013, 68, 95-106.

35. K.E. Washburn, P.T. Callaghan, Journal of Magnetic Resonance 2007, 186, 337.

36. N. Marigheto, L. Venturi, D. Hibberd, K.M. Wright, G. Ferrante, B.P. Hills, Journal of Magnetic Resonance, 2007, 186, 327.

37. R.I. Chelcea, R. Fechete, E. Culea, D.E. Demco and B. Blümich, Journal of Magnetic Resonance, 2009, 196, 178.

38. G.C. Borgia, R.J.S. Brown, and P. Fantazzini, Journal of Magnetic Resonance, 1998, 132, 65. 